\title{
Biological Evaluation of Endophytic Fungus Chaetomium sp. NF15 of Justicia adhatoda L.: A Potential Candidate for Drug Discovery
}

\author{
Nighat Fatima, ${ }^{1,2}$ Usman Mukhtar, ${ }^{1}$ Ihsan-Ul-Haq, ${ }^{3}$ Muneer Ahmed Qazi, ${ }^{1,}{ }^{*}$ Muniba Jadoon, ${ }^{1}$ and Safia \\ Ahmed ${ }^{1}$ \\ ${ }^{1}$ Department of Microbiology, Faculty of Biological Sciences, Quaid-i-Azam University, Islamabad, Pakistan \\ ${ }^{2}$ Department of Biotechnology, Faculty of Biological Sciences, Quaid-i-Azam University, Islamabad, Pakistan \\ ${ }^{3}$ Department of Pharmacy, Faculty of Biological Sciences, Quaid-i-Azam University, Islamabad, Pakistan \\ "Corresponding author: Muneer Ahmed Qazi, Department of Microbiology, Faculty of Biological Sciences, Quaid-i-Azam University, Islamabad 45320, Pakistan. Tel: \\ +92-5190643009, Fax: +92-5190643156, E-mail: qazi_muneer@yahoo.com
}

Received 2015 September 03; Revised 2015 December 28; Accepted 2016 January 29.

\begin{abstract}
Background: The endophytes of medicinal plants, such as Justicia adhatoda L., represent a promising and largely underexplored domain that is considered as a repository of biologically active compounds.

Objectives: The aim of present study was isolation, identification, and biological evaluation of endophytic fungi associated with the J. adhatoda L. plant for the production of antimicrobial, antioxidant, and cytotoxic compounds

Materials and Methods: Endophytic fungi associated with the J. adhatoda L. plant were isolated from healthy plant parts and taxonomically characterized through morphological, microscopic, and 18S rDNA sequencing methods. The screening for bioactive metabolite production was achieved using ethyl acetate extracts, followed by the optimization of different parameters for maximum production of bioactive metabolites. Crude and partially purified extracts were used to determine the antimicrobial, antioxidant, and cytotoxic potential

Results: Out of six endophytic fungal isolates, Chaetomium sp. NF15 showed the most promising biological activity and was selected for detailed study. The crude ethyl acetate extract of NF15 isolate after cultivation under optimized culture conditions showed promising antimicrobial activity, with significant inhibition of the clinical isolates of Staphylococcus aureus (87\%, n=42), Pseudomonas aeruginosa (> 85\%, $\mathrm{n}=41)$, and Candida albicans $(62 \%, \mathrm{n}=24)$.

Conclusions: The present study confirms the notion of selecting endophytic fungi of medicinal plant Justicia for the bioassayguided isolation of its bioactive compounds, and demonstrates that endophytic fungus Chaetomium sp. NF15 could be a potential source of bioactive metabolites
\end{abstract}

Keywords: Antimicrobial, Antioxidant, Endophytes, Chaetomium sp., Justicia adhatoda L.

\section{Background}

Since the discovery of penicillin, microorganisms have been a rich source of novel natural products, offering ease of large-scale cultivation, high potency, and costeffectiveness. The hunt for new antimicrobials is ever increasing, driven by the increased rate of microbial infections and naturally acquired resistance among pathogens (1). Despite the advent of combinatorial chemistry, the natural environment is still the most important source of novel drugs, and has been mined successfully for such agents since long ago (2). Today, researchers emphasize the need to bioprospect for potential leads from relatively diverse and unique environmental settings. Microbial endophytes represent one such unexplored or overlooked group of microorganisms, holding immense potential for the discovery of novel therapeutics (3).

Endophytes are defined as microbes that colonize the living, internal tissues of plants without causing immediate or overt detrimental effects in the host plant (1). Almost all vascular plant species examined to date act as hosts to either endophytic fungi or bacteria (4). The relationship between endophytes and their host plants is mutualistic in nature, resulting in a survival benefit for both of the partners. In fact, endophytes act as chemical synthesizers inside the host plant, producing a plethora of bioactive secondary metabolites $(3,5)$. Studies have reported the isolation and characterization of unique structures, such as alkaloids, terpenoids, and flavonoids, from these ubiquitous organisms (6). These bioactive metabolites accentuate the biotechnological importance of endophytes, as they are useful for a wide range of applications, including antibiotics, anticancer compounds, antioxidants, immunosuppressants, and biological control agents (7, 8). As they have been found in almost all plant species studied to date, there is ample opportunity to unearth novel en-

Copyright (c) 2016, Ahvaz Jundishapur University of Medical Sciences. This is an open-access article distributed under the terms of the Creative Commons Attribution-NonCommercial 4.0 International License (http://creativecommons.org/licenses/by-nc/4.0/) which permits copy and redistribute the material just in noncommercial usages, provided the original work is properly cited. 
dophytic microorganisms with significant therapeutic potential (9).

Medicinal plants in particular are considered a huge repository of endophytes with pharmaceutical importance (10). Various fungal endophytic strains (e.g., Penicillium, Alternaria, Mucor) have been isolated from plants. Chaetomium species are among the biologically active endophytes. Chaetomium is reported to be involved in the production of chemically diverse metabolites, such as chaetoglobosins, anthraquinones, xanthones, depsidones, terpenoids, and steroids (11). These metabolites possess anticancer, antimicrobial, antioxidant, and cytotoxic properties. Therefore, in the current study, we selected the Chaetomium strain NF15 for optimization.

\section{Objectives}

The objective of the present study was to isolate and characterize the endophytic fungi associated with the J. adhatoda L. plant, and to investigate their antimicrobial, antioxidant, and cytotoxic potential.

\section{Materials and Methods}

Potato dextrose agar (PDA) and sabouraud dextrose agar (SDB) were purchased from Oxoid UK. Dimethyl sulfoxide (DMSO) and 2,2-diphenyl-1-picrylhydrazyl (DPPH) were purchased from Merck USA. All other chemicals, including cetyltrimethylammonium bromide (CTAB), $\mathrm{Ca}\left(\mathrm{NO}_{3}\right)_{2} 4 \mathrm{H}_{2} \mathrm{O}, \mathrm{KNO}_{3}, \mathrm{MgSO}_{4} 7 \mathrm{H}_{2} \mathrm{O}, \mathrm{KCl}, \mathrm{NaH}_{2} \mathrm{PO}_{4}, \mathrm{FeCl}_{3}$, and $\mathrm{MnSO}_{4}$, were purchased from Merck USA and SigmaAldrich. The spectrophotometer was a model 8354 from Agilent Technologies (Germany).

\subsection{Collection of Plant Samples}

Healthy and mature plants of Justicia were selected for sample collection. The plant leaves were randomly collected and brought to the laboratory under sterile conditions. The plant was identified as J. adhatoda by Prof. Dr. Mir Ajab Khan at the department of plant sciences, Quaidi-Azam university, Islamabad.

\subsection{Isolation of Endophytic Fungi}

Isolation of fungal endophytes was carried out with the surface sterilization method (12). The plant material was thoroughly washed with distilled water, followed by treatment with $100 \%$ ethanol for 2 min and 5\% sodium hypochlorite for $5 \mathrm{~min}$. The material was again rinsed with distilled water, and finally blotted with sterile blotting papers. After sterilization, the samples were cut into pieces measuring 2 - $6 \mathrm{~mm}$, then placed on water agar and
PDA plates supplemented with penicillin G (100 units) and streptomycin $(100 \mu \mathrm{g} / \mathrm{mL})$. All of the plates were incubated at $28^{\circ} \mathrm{C}$ until growth initiation (13). The hyphal tips that emerged as a result of incubation were subcultured for purification, and the isolated pure cultures were maintained on PDA for further studies.

\subsection{Cultivation and Extraction of Fungal Metabolites}

The fungal endophytes isolated from the Justicia plant were cultured on SDA plates for liquid fermentation. Three pieces $\left(0.5 \times 0.5 \mathrm{~cm}^{2}\right)$ of mycelial agar plugs were inoculated into a 500-mL Erlenmeyer flask containing $200 \mathrm{~mL}$ of SDB, then run on a shaker incubator (New Brunswick, Canada) at $28^{\circ} \mathrm{C}$ for 14 days. After incubation, the fermentation broth was filtered to remove the mycelial mass, and the filtrate was extracted three times with equal volumes of ethyl acetate. The organic solvent was pooled and concentrated to dryness using a rotary evaporator to obtain the crude ethyl acetate extract. A stock solution for all of the extracts was prepared by suspending the crude extracts in DMSO at a ratio of $10 \mathrm{mg} / \mathrm{mL}$, which were then screened for antimicrobial activity against selected pathogens.

\subsection{Biological Screening}

The crude ethyl acetate extract obtained after culturing all fungal isolates in SDB medium was used for biological screening with the following assays.

\subsubsection{Antibacterial Assay}

The crude ethyl acetate extracts of all fungal endophytes were tested against multidrug resistant (MDR) strains of Staphylococcus aureus, Micrococcus luteus, Escherichia coli, and Pseudomonas aeruginosa. The antibacterial potential of the samples was determined by using the agar well diffusion assay (14). Test bacterial strains of comparable turbidity with $0.5 \%$ McFarland's solution were used to create lawn on nutrient agar plates. Wells with 8mm diameters were made with a sterile metallic borer, and $100 \mu \mathrm{L}$ of each extract $(10 \mathrm{mg} / \mathrm{mL})$ was poured into the respective wells with a micropipette. Pure DMSO was used as a negative control and tetracycline was used as a positive control. The plates were incubated at $37^{\circ} \mathrm{C}$. After 24 hours of incubation, the diameter of each zone of inhibition was measured in millimeters. The assay was performed in triplicate for each strain.

\subsubsection{Antifungal Assay}

The antifungal assay was performed with the agar well diffusion method (15). The fungal test cultures used in this assay were Aspergillus niger, A. terreus, and Candida albicans. 
PDA was used as the growth medium for the fungal test cultures. Inoculum suspension was adjusted with a hemocytometer (Neubauer Chamber; Merck, S.A., Madrid, Spain). Each test isolate (an aliquot of spore suspension at $1 \times 10^{8}$ spores $/ \mathrm{mL}$ ) was spread evenly onto the plates with sterile glass rods, and wells ( $6 \mathrm{~mm}$ ) were created at appropriate distances. An aliquot of $50 \mu \mathrm{L}$ of crude ethyl acetate (10 $\mathrm{mg} / \mathrm{mL}$ ) was dispensed carefully into the wells. The plates were incubated at $27^{\circ} \mathrm{C}$, and the results were noted after 24 hours for the Candida species and after 48 hours for the other fungi. Pure DMSO and Nystatin were used as negative and positive controls, respectively. Antifungal activity was expressed as the diameter of the zone of inhibition, measured in millimeters.

\subsection{Molecular Characterization of the Selected Fungal Isolate}

The selected fungal isolate was further characterized using the molecular $18 \mathrm{~S}$ rDNA sequencing methodology for complete identification. The genomic DNA was obtained from freshly grown mycelia using the standard CTAB (cetyl trimethylammonium bromide, Merck USA) DNA extraction method as described by Qazi et al. (16). The universal primers ITS1 (5'-TCCGTAGGTGAACCTGCGG-3') and ITS4 (5'-TCCTCCGCTTATTGATATGC-3') were used for amplification of the fungal internal transcribed spacer (ITS) region of $18 \mathrm{~S}$ rDNA (17). The phylogenetic tree was constructed through alignment of the sequences of the NCBI GenBank data using molecular and evolutionary genetics analysis (MEGA) software (version 4.0). The nucleotide sequence of the NF15 strain was submitted to GenBank and an accession number was obtained.

\subsection{Antioxidant Assay}

The free radical scavenging activity was measured with the 2,2-diphenyl-1-picrylhydrazyl (DPPH, Merck USA) assay (18). The assay was performed on a 96-well plate containing $5 \mu \mathrm{L}$ of test solution and $95 \mu \mathrm{L}$ of DPPH solutions (316 $\mu \mathrm{M}$ in methanol). The maximum concentration tested was $200 \mu \mathrm{g} / \mathrm{mL}$. The plates were incubated at $37^{\circ} \mathrm{C}$ for $30 \mathrm{~min}-$ utes, and absorbance was measured at $515 \mathrm{~nm}$ using a microplate reader. The antioxidant potential was calculated as percent scavenging effect with Equation 1. Ascorbic acid and pure DMSO were used as positive and negative controls, respectively. The assay was performed in triplicate for all fractions.

Scaving effect $(\%)=\frac{A_{c}-A_{s}}{A_{s}} \times 100$

where $\mathrm{A}_{\mathrm{c}}=$ absorbance of control and $\mathrm{A}_{\mathrm{s}}=$ absorbance of test sample.

\subsection{Optimization of Media and Culture Conditions}

\subsubsection{Media Selection}

The selected NF15 isolate was cultured in four different media: potato dextrose broth (PDB), sabouraud dextrose broth (SDB), yeast malt peptone glucose (YMG) broth, and mineral salt (M4) medium. The YMG medium contained $4 \mathrm{~g} / \mathrm{L}$ of yeast extract, $10 \mathrm{~g} / \mathrm{L}$ of malt extract, and 10 $\mathrm{g} / \mathrm{L}$ of glucose, while the M4 medium contained $3 \mathrm{~g} / \mathrm{L}$ of sucrose, $0.25 \mathrm{~g} / \mathrm{L}$ of yeast extract, $5 \mathrm{~g} / \mathrm{L}$ of $\mathrm{C}_{4} \mathrm{H}_{12} \mathrm{~N}_{2} \mathrm{O}_{6}, 0.5 \mathrm{~g} / \mathrm{L}$ of $\mathrm{Ca}\left(\mathrm{NO}_{3}\right)_{24} \mathrm{H}_{2} \mathrm{O}, 0.08 \mathrm{~g} / \mathrm{L}$ of $\mathrm{KNO}_{3}, 0.36 \mathrm{~g} / \mathrm{L}$ of $\mathrm{MgSO}_{4} 7 \mathrm{H}_{2} \mathrm{O}$, $0.06 \mathrm{~g} / \mathrm{L}$ of $\mathrm{KCl}, 0.02 \mathrm{~g} / \mathrm{L}$ of $\mathrm{NaH}_{2} \mathrm{PO}_{4}, 0.002 \mathrm{~g} / \mathrm{L}$ of $\mathrm{FeCl}_{3}$, $0.005 \mathrm{~g} / \mathrm{L}$ of $\mathrm{MnSO}_{4}, 0.003 \mathrm{~g} / \mathrm{L}$ of $\mathrm{ZnSO}_{4} 7 \mathrm{H}_{2} \mathrm{O}, 0.0014 \mathrm{~g} / \mathrm{L}$ of $\mathrm{H}_{3} \mathrm{BO}_{3}$, and $0.0007 \mathrm{~g} / \mathrm{L}$ of $\mathrm{KI}$. The YMG and M4 media were used for fermentation.

The shake flasks $(250 \mathrm{~mL})$ containing $100 \mathrm{~mL}$ of each medium broth were inoculated with three mycelial agar plugs $\left(0.5 \times 0.5 \mathrm{~cm}^{2}\right)$ of freshly grown pure fungal culture, and incubated at $30^{\circ} \mathrm{C}$ at $150 \mathrm{rpm}$ for 14 days. After cultivation, to screen the most ideal medium for bioactive metabolite production, the antibacterial activity of the cell-free supernatant of each fermentation medium was checked against the indicator strain P. aeruginosa.

\subsubsection{Optimization of Operational Parameters}

The different parameters that affected the growth and production of the required metabolites by NF15, such as incubation time (7, 14, and 21 days), $\mathrm{pH}(4,5,6,7$, and 8$)$, temperature $\left(20,25,30\right.$, and $\left.35^{\circ} \mathrm{C}\right)$, and agitation $(0,100,120$, and $150 \mathrm{rpm}$ ), were optimized one parameter at a time (16). The optimization was carried out using SDB as the production medium. Three 250-mL Erlenmeyer flasks, each containing $100 \mathrm{~mL}$ of SDB, were inoculated with two pieces $(0.5$ $\times 0.5 \mathrm{~cm}^{2}$ ) of mycelial agar from a 5-day-old culture plates for each parameter. Cell-free supernatant from all of the experimental flasks was tested for activity against P. aeruginosa.

\subsubsection{Optimization of Additional Salts}

At optimum culture conditions, the effect of the addition of $\mathrm{KH}_{2} \mathrm{PO}_{4}, \mathrm{KNO}_{3}$, and $\mathrm{NaCl}$ into the production medium at varying concentrations $(2,4,6$, and $8 \mathrm{~g} / \mathrm{L})$ was tested. The different concentrations of these nutrients were added to four flasks containing $100 \mathrm{~mL}$ of SDB, which were then incubated for 14 days at $30^{\circ} \mathrm{C}$ and $150 \mathrm{rpm}$, after inoculation with fungal endophytes. After incubation, the cell-free supernatant was tested against the $P$. aeruginosa strain.

3.8. Production, Extraction, and Fractionation of the Bioactive Metabolites Under Optimized Conditions

The selected endophytic fungal isolate was cultured under optimized conditions in $500 \mathrm{~mL}$ of SDB, inoculated 
with nine mycelial plugs, and incubated $\left(30^{\circ} \mathrm{C}, 150 \mathrm{rpm}\right)$ for 14 days. After incubation, the cell-free broth was extracted and fractionated as described in the scheme shown in Figure 1. All of the chemicals were purchased from Sigma-Aldrich. The extracts were pooled and subjected to partial purification with the solvent-solvent partitioning process (18). All of the fractions were concentrated to dryness using a rotary evaporator (Heidolph LabTech, Germany) and tested for their antibacterial and antioxidant activities.

\section{Results}

4.1. Isolation, Screening, and Preliminary Identification of Endophytic Fungi

A total of six isolates (NF12, NF13, NF14, NF15, NF16, and NF17) were obtained from healthy plant parts (Figure 2). Most of the isolates produced only sterile mycelium, while NF15 also exhibited spore production. The morphological features showed that isolate NF15 belongs to the genus Chaetomium; however, further confirmation using molecular techniques was required. screening results for the antimicrobial metabolites revealed that three isolates, NF14, NF15, and NF16, displayed considerable antibacterial (Table 1) and antifungal (Table 2 ) activities against $S$. aureus and $P$. aeruginosa (inhibitory zone, 9 - $15 \mathrm{~mm}$ ) and C. albicans (inhibitory zone, $9-13 \mathrm{~mm}$ ). The endophytic fungal isolate NF15, expressing the maximum antimicrobial activity, was then selected for further investigation.

\subsection{Molecular Characterization of Fungal Isolate NF15}

The fungal isolate NF15 was characterized on the basis of $18 \mathrm{~S}$ rDNA gene sequencing. Figure 3 illustrates the phylogenetic tree showing the evolutionary relationship of isolate NF15 with other closely related taxa. It was found that this isolate shared $100 \%$ similarity with Chaetomium sp. ATT106 [GenBank: HQ607837.1]. Therefore, the isolate was identified and named Chaetomium sp. NF15.

\subsection{Optimization of Culture Conditions for NF15}

\subsubsection{Effect of Incubation Time}

The effect of incubation time on the growth and production of antimicrobial metabolites was studied by incubating the inoculated flasks for 7,14, and 21 days. Maximum antimicrobial activity was observed after 14 days of incubation, when the zone of inhibition against the selected test strain was $17 \mathrm{~mm}$, with a biomass $9.5 \mathrm{~g} / \mathrm{L}$. An increased incubation time of longer than 14 days resulted in increased biomass and decreased activity (Figure 4A).

\subsubsection{Effect of Agitation Speed}

To determine the influence of the agitation speed, metabolite production was carried out at 0,100,120, and $150 \mathrm{rpm}$. Antimicrobial activity was observed at all agitation speeds. However, the best activity was observed at an agitation speed of $150 \mathrm{rpm}$, with a zone of inhibition measuring $18 \mathrm{~mm}$. A minimum zone of inhibition of $8 \mathrm{~mm}$ was observed under static conditions. An increased agitation speed resulted in a gradual increase in biomass, with a maximum value at $150 \mathrm{rpm}$ (Figure 4B).

\subsubsection{Effect of $p H$}

The selected isolate showed variations in antimicrobial activity when subjected to different $\mathrm{pH}$ values. Maximum activity was found at a pH value of 6 , with a zone of inhibition of $20 \mathrm{~mm}$ and a biomass of $7.94 \mathrm{~g} / \mathrm{L}$. The isolate did not exhibit any growth or activity at a $\mathrm{pH}$ of 4 , while consistent growth without any measureable activity was observed at a $\mathrm{pH}$ of 8 (Figure $4 \mathrm{C}$ ).

\subsubsection{Effect of Temperature}

The effect of temperature on the production of antimicrobial metabolites by NF15 revealed that the isolate remained active at all of the rendered temperature values (Figure 4D). However, the most significant activity was observed at $30^{\circ} \mathrm{C}$, where the zone of inhibition and biomass were noted to be $28 \mathrm{~mm}$ and $15.1 \mathrm{~g} / \mathrm{L}$, respectively.

\subsubsection{Effect of Cultivation Media and Salts}

After optimization of operational parameters, the effects of different growth media and salt concentrations on the activity and biomass production of NF15 were evaluated. Although all of the media supported the growth and production of antimicrobial metabolites by NF15 (Figure $5 \mathrm{~A})$, the maximum zone of inhibition ( $16 \mathrm{~mm}$ ) and biomass production $(8.2 \mathrm{~g} / \mathrm{L})$ were achieved when SDB medium was used. Similarly, three different salts $\left(\mathrm{NaCl}, \mathrm{KH}_{2} \mathrm{PO}_{4}\right.$, and $\mathrm{KNO}_{3}$ ) were selected to determine the effects of sodium, phosphate, and nitrogen contents on NF15. Different concentrations of $\mathrm{NaCl}(2,4,6$, and $8 \mathrm{~g} / \mathrm{L})$ were applied, and it was observed that growth, as well as bioactive metabolite production, was enhanced up to $4 \mathrm{~g} / \mathrm{L}$ (Figure 5B -5D). It was observed that the highest activity was at a concentration of $2 \mathrm{~g} / \mathrm{L}$ (zone of inhibition, $9.2 \mathrm{~mm}$ ), whereas beyond this concentration, the activity was diminished. In addition, different concentrations of potassium dihydrogen phosphate $(2,4,6$, and $8 \mathrm{~g} / \mathrm{L}, \mathrm{w} / \mathrm{v})$ were evaluated. A high phosphate concentration suppressed growth and bioactive metabolite production. Maximum activity was observed at a concentration of $2 \mathrm{~g} / \mathrm{L}$, with a zone of inhibition of $10.5 \mathrm{~mm}$ and a biomass of $9.4 \mathrm{~g} / \mathrm{L}(\mathrm{w} / \mathrm{v})$. In the case of nitrogen salt, the highest activity was observed at a 


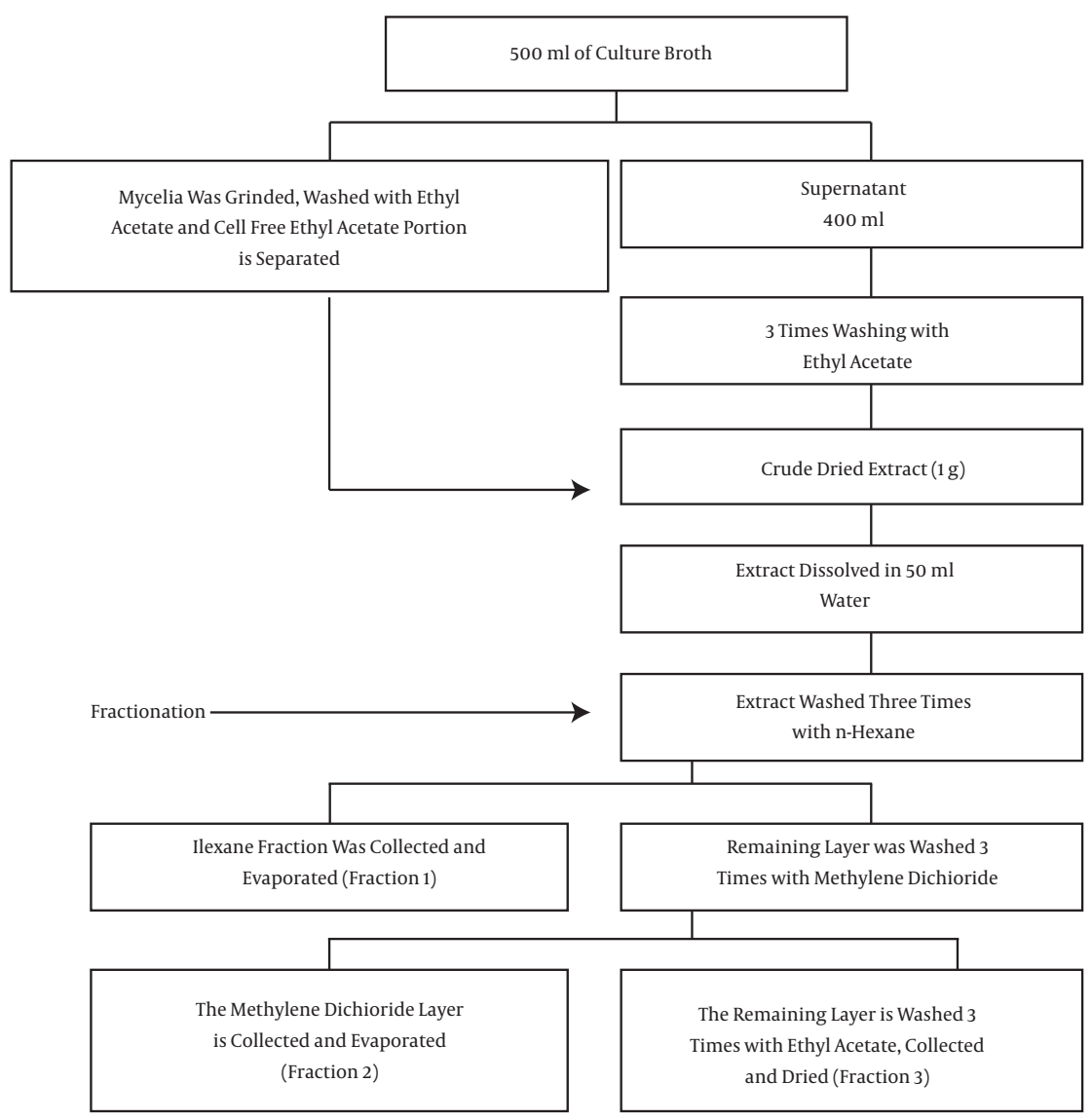

Figure 1. Schematic Representation of Cultivation, Extraction, and Fractionation of the Bioactive Compounds of Chaetomium sp. NF15

Table 1. Screening for Antibacterial Metabolite Production by Different Endophytic Fungal Isolates of Justicia adhatoda

\begin{tabular}{|c|c|c|c|c|}
\hline \multirow[t]{2}{*}{ Fungal Strain } & \multicolumn{4}{|c|}{ Antibacterial Activity: Zone of Inhibition \pm SD (Homogenous Group Based on LSD at $\mathrm{P}<0.05)$} \\
\hline & S.aureus & E.coli & M.luteus & P.aeruginosa \\
\hline NF12 & $0.0 \pm 0.0(\mathrm{~d})$ & $0.0 \pm 0.0(\mathrm{c})$ & $0.0 \pm 0.0(\mathrm{c})$ & $0.0 \pm 0.0(\mathrm{c})$ \\
\hline NF13 & $0.0 \pm 0.0(\mathrm{~d})$ & $0.0 \pm 0.0(\mathrm{c})$ & $0.0 \pm 0.0(\mathrm{c})$ & $0.0 \pm 0.0(c)$ \\
\hline NF14 & $13.9 \pm 1.2(b)$ & $0.0 \pm 0.0(\mathrm{c})$ & $0.0 \pm 0.0(\mathrm{c})$ & $9.8 \pm 0.6(b)$ \\
\hline NF16 & $10.1 \pm 0.5(\mathrm{c})$ & $0.0 \pm 0.0(\mathrm{c})$ & $0.0 \pm 0.0(\mathrm{c})$ & $9.5 \pm 0.3(b)$ \\
\hline NF17 & $0.0 \pm 0.0(\mathrm{~d})$ & $0.0 \pm 0.0(c)$ & $0.0 \pm 0.0(\mathrm{c})$ & $0.0 \pm 0.0(\mathrm{c})$ \\
\hline Tetracycline $30 \mu \mathrm{g} /$ well & $20.1 \pm 1.0(a)$ & $21.2 \pm 1.6(\mathrm{a})$ & $22.3 \pm 1.6(a)$ & $14.5 \pm 2.1(\mathrm{a})$ \\
\hline DMSO & $0.0 \pm 0.0(\mathrm{~d})$ & $0.0 \pm 0.0(\mathrm{c})$ & $0.0 \pm 0.0(\mathrm{c})$ & $0.0 \pm 0.0(\mathrm{c})$ \\
\hline
\end{tabular}

Abbrviations: DMSO, dimethylsulfoxide; LSD, least significant difference; SD, standard deviation.

${ }^{\mathrm{a}}$ The values are represented as the mean \pm standard deviation among triplicate experiments. Values followed by the same alphabetic letter do not differ significantly at a $95 \%$ probability $(\mathrm{P}<0.05)$

concentration of $4 \mathrm{~g} / \mathrm{L}$, at which the zone of inhibition and biomass were $22 \mathrm{~mm}$ and $11.1 \mathrm{~g} / \mathrm{L}$, respectively (Figure 5B -
5D). No activity was observed at $8 \mathrm{~g} / \mathrm{L}$, and a reduction in biomass was also noted at this concentration. 


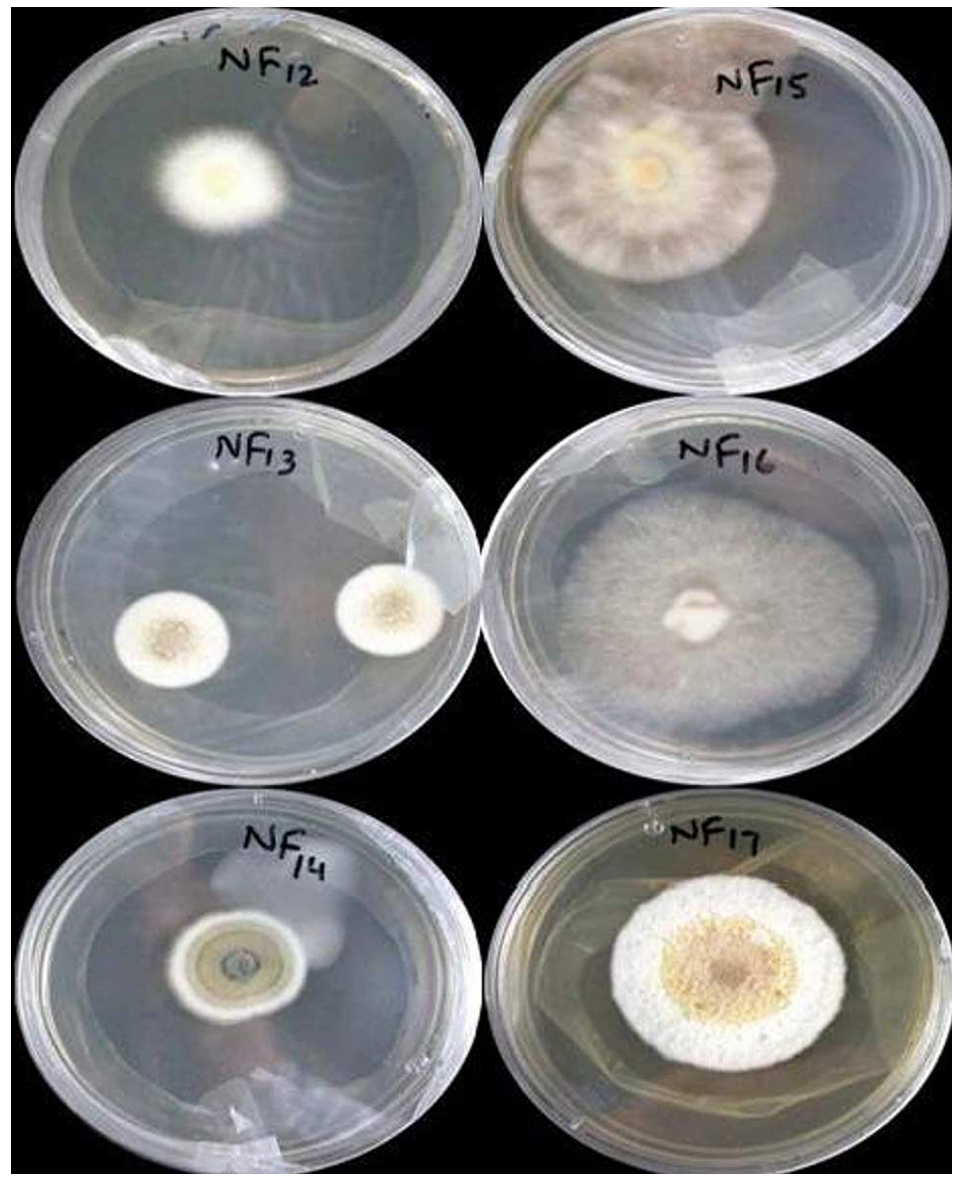

Figure 2. Morphological Features and Growth Patterns of Endophytic Fungal Isolates of Justicia adhatoda L. Plant on SDA Plates after 14 days of Incubation at $28^{\circ} \mathrm{C}$

Table 2. Screening for Antifungal Metabolite Production by Different Endophytic Fungal Isolates of Justicia adhatoda

\begin{tabular}{|c|c|c|c|}
\hline \multirow[t]{2}{*}{ Fungal Strain } & \multicolumn{3}{|c|}{ Antifungal Activity: Zone of Inhibition $\pm S D($ Homogenous Group Based on LSD at $P<0.05)$} \\
\hline & A. niger & A.terreus & C.albicans \\
\hline NF12 & $0.0 \pm 0.0(\mathrm{c})$ & $0.0 \pm 0.0(\mathrm{~b})$ & $0.0 \pm 0.0(\mathrm{~d})$ \\
\hline NF13 & $0.0 \pm 0.0(\mathrm{c})$ & $0.0 \pm 0.0(\mathrm{~b})$ & $0.0 \pm 0.0(\mathrm{~d})$ \\
\hline NF14 & $0.0 \pm 0.0(\mathrm{c})$ & $0.0 \pm 0.0(\mathrm{~b})$ & $9.9 \pm 0.3(c)$ \\
\hline NF16 & $0.0 \pm 0.0(\mathrm{c})$ & $0.0 \pm 0.0(\mathrm{~b})$ & $9.7 \pm 0.4(\mathrm{c})$ \\
\hline NF17 & $0.0 \pm 0.0(\mathrm{c})$ & $0.0 \pm 0.0(\mathrm{~b})$ & $0.0 \pm 0.0(\mathrm{~d})$ \\
\hline Nystatin $10 \mu \mathrm{g} /$ well & $24.2 \pm 1.6(\mathrm{a})$ & $21.5 \pm 1.0(\mathrm{a})$ & $19.5 \pm 1.2(\mathrm{a})$ \\
\hline DMSO & $0.0 \pm 0.0(\mathrm{c})$ & $0.0 \pm 0.0(b)$ & $0.0 \pm 0.0(\mathrm{~d})$ \\
\hline
\end{tabular}

Abbrviations: LSD, least significant difference; SD, standard deviation.

${ }^{\mathrm{a}}$ The values are represented as the mean \pm standard deviation among triplicate experiments. The values followed by the same alphabetic letter do not differ significantly at a $95 \%$ probability $(\mathrm{P}<0.05)$.

\subsection{Biological Screening}

4.4.1. Antimicrobial Assays

Antibacterial activity was present only in the ethyl acetate fraction. A comparison of crude extract before and af- ter optimization and fractionation was made by testing the

Jundishapur J Microbiol. 2016; 9(6):e29978. 


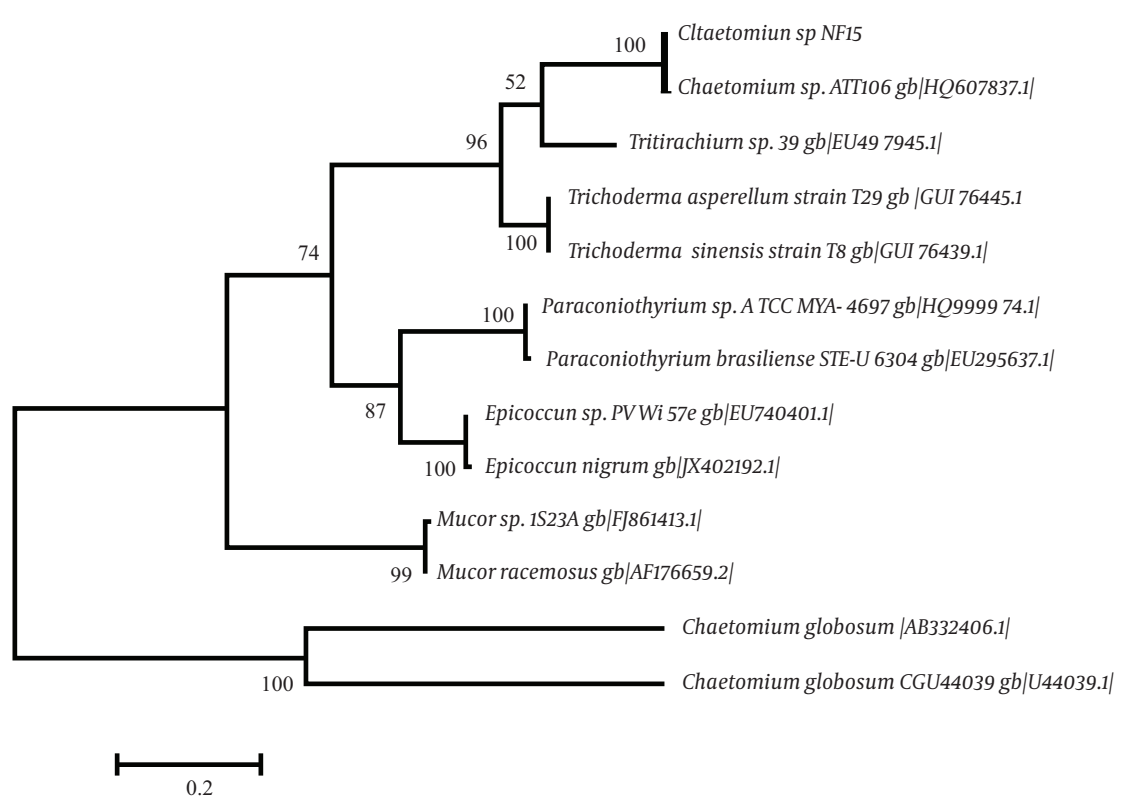

The evolutionary history was inferred by using the maximum likelihood method, based on the Tamura-Nei model [1]. The tree with the highest log likelihood (-2629.5593) is shown. The percentage of trees in which the associated taxa are clustered together is shown next to the branches. The initial tree(s) for the heuristic search were obtained automatically by applying neighbor-joining and BioNJ algorithms to a matrix of pairwise distances estimated using the maximum composite likelihood (MCL) approach, and then selecting the topology with the superior log likelihood value. The tree is drawn to scale, with branch lengths measured in the number of substitutions per site. The analysis involved 13 nucleotide sequences. The included codon positions were $1 s t+2 n d+3 r d+$ Noncoding. All positions containing gaps and missing data were eliminated. There were a total of 318 positions in the final dataset. Evolutionary analyses were conducted in MEGA6 [2].

samples against $P$. aeruginosa. The results showed a significant increase in the zone of inhibition of the ethyl acetate fraction. When tested against MDR strains, $85 \%$ of 41 samples of MDR strains of P. aeruginosa and $87 \%$ of 42 S. aureus (MRSA) strains were sensitive to the partially purified ethyl acetate fraction (Figure 6). Only $24 \%$ of the Enterococci isolates were sensitive, while no activity was observed against E. coli. A significant zone of inhibition was formed against tobramycin-resistant $P$. aeruginosa. With regard to antifungal activity, $65 \%$ of $C$. albicans isolates were sensitive to partially purified ethyl acetate (Figure 6).

\subsubsection{Antioxidant Assay}

Antioxidant activity was analyzed using two different concentrations: 5 and $10 \mu \mathrm{g} / \mathrm{mL}$. Both samples showed a strong scavenging effect. Increased concentrations led to increased antioxidant activity, with a 79\% scavenging effect at $10 \mu \mathrm{g} / \mathrm{mL}$ (Table 3).

\section{Discussion}

The increasing prevalence of drug-resistant bacteria necessitates the need to search for novel sources of antibiotics. In this quest, endophytic microorganisms have been identified as a rich source due to the highly competitive environment in which they survive (19). The chemical variety of biologically active secondary metabolites from endophytic fungi has led to further research on these organisms (20).

In the present study, we successfully isolated endophytic fungi from J. adhatoda. This plant has ethnobotanical importance in folklore medicine $(21,22)$. It is likely that some medicinal attributes of the Justicia plant could be transferred to endophytes through horizontal gene transfer. This is affirmed by the present study, in which the isolates showed promising antimicrobial and antifungal activity. There are some recent reports about the biological activities of endophytes of Justicia species $(23,24)$. Gangadevi and Muthumary (25) reported the isolation of Colletotrichum gloeosporioides from J. gendarussa, and their antimicrobial activities. After an initial antimicrobial screening, we selected one isolate, NF15, for identification, extraction, and partial purification of bioactive secondary metabolites.

Molecular identification of NF15 showed that it resembles Chaetomium sp. (17). Several types of bioactive compounds have been reported from this unique fungal specie. Chaetomium species are able to produce antimicro- 
A

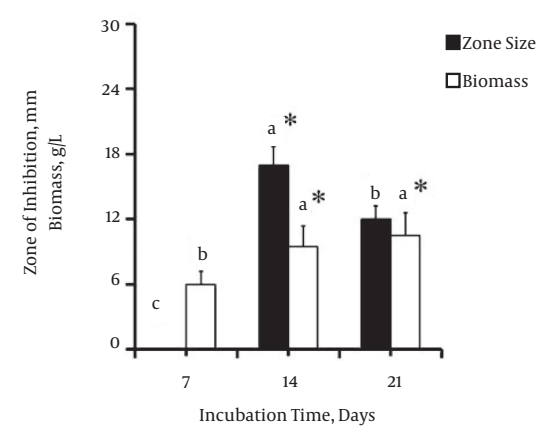

C

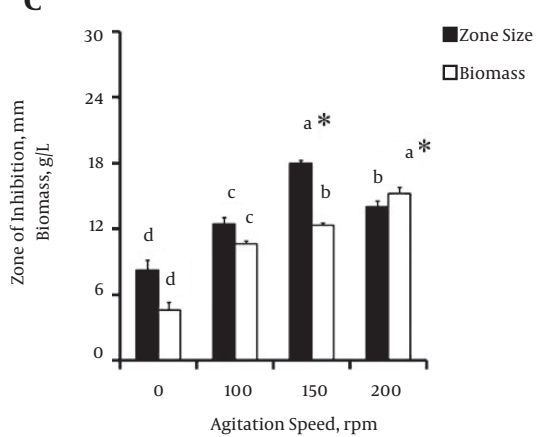

B

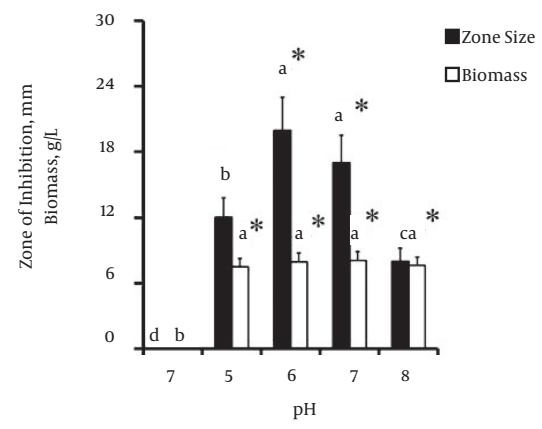

D

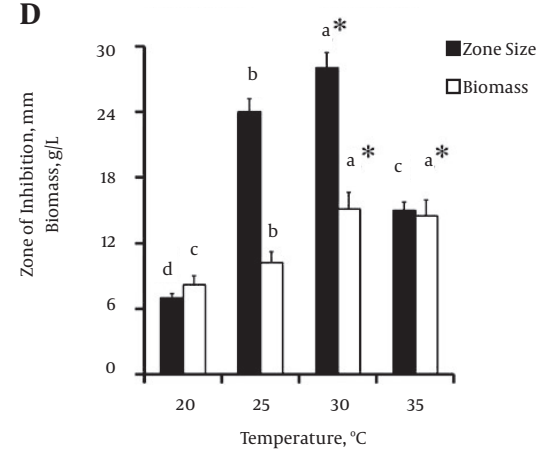

A, effect of incubation time in days, B, effect of agitation speed (rpm) of the shaking incubator during the incubation period, $\mathrm{C}$, effect of the pH of the production medium, and $\mathrm{D}$, effect of incubation temperature $\left({ }^{\circ} \mathrm{C}\right)$ at four different levels. All experiments were performed in triplicate and the values are represented as the mean of three observations. The error bars indicate the standard deviation among treatments. The bars tagged with same alphabetic letter do not differ significantly at a $95 \%$ confidence level, while letters followed by the asterisk symbol $\left({ }^{*}\right)$ denote significantly higher values $(\mathrm{P}<0.05)$.

Table 3. Free Radical Scavenging Activity (RSA) of the Partially Purified Product (Ethyl Acetate Fraction) of NF15 Isolate.

\begin{tabular}{|c|c|c|c|c|}
\hline \multirow[t]{2}{*}{ Reaction Time, min } & \multicolumn{2}{|c|}{ At $5 \mu \mathrm{g} / \mathrm{mL}$} & \multicolumn{2}{|c|}{ At $10 \mu \mathrm{g} / \mathrm{mL}$} \\
\hline & O.D. $517 \mathrm{~nm}$ & RSA, \% & O.D. $517 \mathrm{~nm}$ & RSA, \% \\
\hline $\mathbf{0}$ & $0.58 \pm 0.03$ & - & $0.93 \pm 0.05$ & - \\
\hline 10 & $0.19 \pm 0.01$ & $67.0 \pm 2.35$ & $0.21 \pm 0.01$ & $77.0 \pm 2.85$ \\
\hline 30 & $0.15 \pm 0.01$ & $74.1 \pm 3.74$ & $0.19 \pm 0.01$ & $79.0 \pm 3.95$ \\
\hline
\end{tabular}

bial, cytotoxic, anticancer, antimalarial, and antioxidant compounds with chemical diversity $(11,26)$. Chaetomium species have been used to produce of a plethora of pharmacologically active metabolites, including chaetoglobosins, cytoglobins, anthraquinones, steroids, azaphilones, alkaloids, and depsidones $(11,26,27)$. On the basis of previous reports, Chaetomium sp. NF15 was selected for detailed study of its bioactive metabolites by adopting the reported methods with slight modifications (16). The initial screen- ing was done using a simple method of fermentation and extraction, but a further detailed optimization study was also performed.

Endophytes undergo complex interactions with plants, and nutrients and culture conditions play an important role in the onset and intensity of secondary metabolism (28). In order to achieve high product yields from the selected NF15 isolate, it was necessary to design a proper production medium for an efficient fermentation 
A

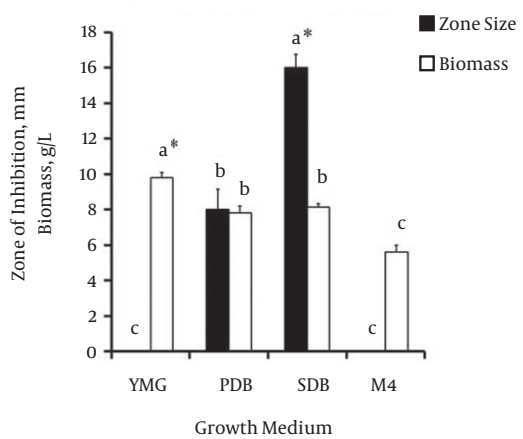

C

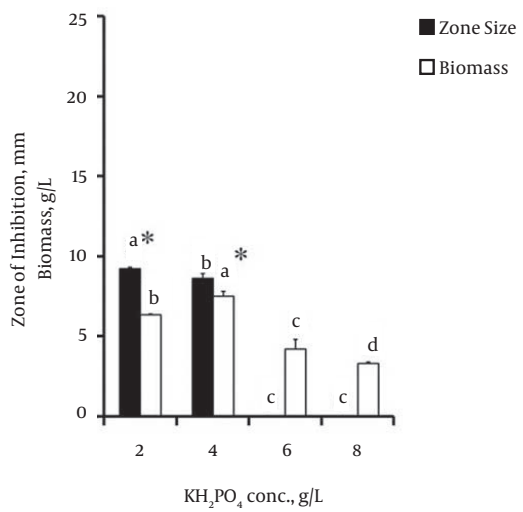

B

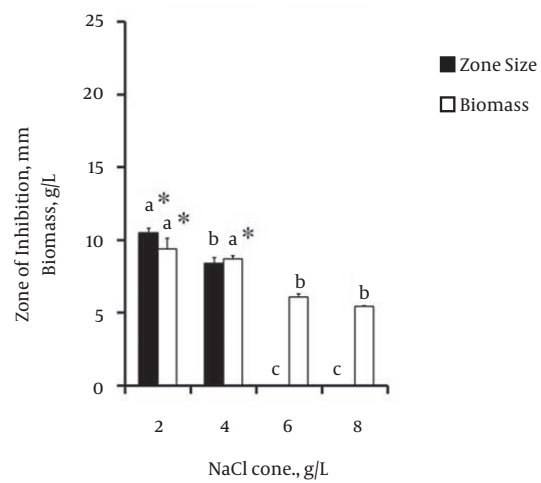

D

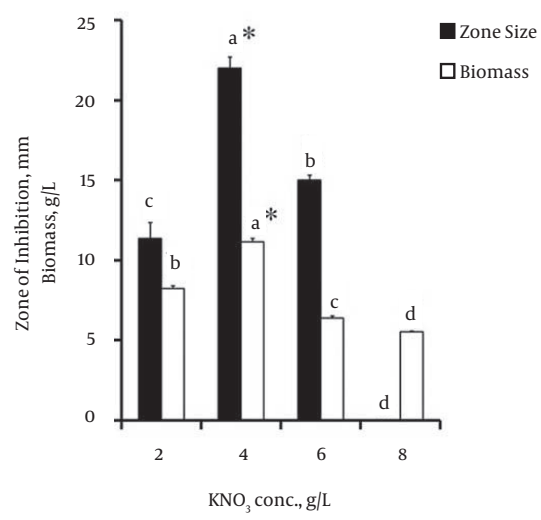

Figure 5. A, Effect of growth media on the growth and production of antimicrobial metabolites from endophytic fungal isolate NF15. The error bars indicate the standard deviation among the treatments. The bars tagged with the same alphabetic letter do not differ significantly at a $95 \%$ confidence level, while the letters followed by the asterisk symbol $\left({ }^{*}\right)$ denote significantly higher values $(\mathrm{P}<0.05)$. B - D, Effect of different salts at varying concentrations on the growth and production of antimicrobial metabolites from endophytic fungal isolate $\mathrm{NF} 15$. B, Effect of $\mathrm{NaCl}$, C, effect of $\mathrm{KH}_{2} \mathrm{PO}_{4}$, and D, effect of $\mathrm{KNO}_{3}$. The error bars indicate the standard deviation among treatments. The bars tagged with the same alphabetic letter do not differ significantly at a $95 \%$ confidence level, while the letters followed by the asterisk symbol $\left({ }^{*}\right)$ denote significantly higher values $(\mathrm{P}<0.05)$.

process $(16,29)$. Fungal isolates showed better growth and antimicrobial potential in SDB compared to the other media. This is in accordance with Gogoi et al. (30), who reported improved metabolite production in the presence of dextrose. Maximum production of secondary metabolites was observed after 14 days of incubation, which agrees with previous reports $(30,31)$.

The isolated strain NF15 was able to grow and produce metabolites at all of the pH values tested; however, maximum metabolite production was at a $\mathrm{pH}$ of 6 , as previously reported (30-32). Temperature optimization results identified antimicrobial activity at $30^{\circ} \mathrm{C}$, which is reported as a suitable temperature for metabolite production (33). Agitation is another factor that affects cell growth and the production of secondary metabolites. We analyzed the effects of four different agitation speeds, and found that the isolates produced antimicrobial metabolites at all speeds.
However, the highest activity was at $150 \mathrm{rpm}$, which correlates with previous findings that the aeration rate affects metabolite production (34).

Optimal nutrients and culture conditions could help in obtaining the maximum yield of the target metabolite and diminishing unwanted metabolites (35). After optimizing the abiotic factors, the effects of different medium components and their concentrations were determined (36). Fungus showed good antimicrobial potential at low salt concentrations. Sodium chloride concentrations up to $4 \mathrm{~g} / \mathrm{L}$ and $\mathrm{KNO}_{3}$ up to $6 \mathrm{~g} / \mathrm{L}$ had positive effects on bioactive metabolite production by NF15 (37). However, phosphate confers negative effects if the concentration is increased beyond $2 \mathrm{~g} / \mathrm{L}(38,39)$.

The selected NF15 isolate was cultured under optimum conditions for 14 days at $30^{\circ} \mathrm{C}$ and $150 \mathrm{rpm}$ in $500 \mathrm{~mL}$ of medium, and the crude extract was used for fractionation. 
Figure 6. Sensitivity Pattern of Partially Purified Product (Ethyl Acetate Fraction) Against MDR Isolates

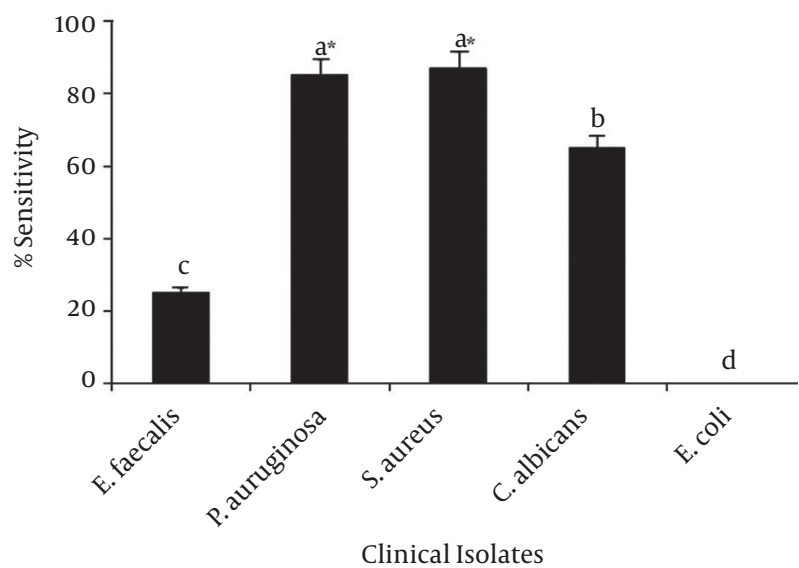

The error bars indicate the standard deviation among the treatments. The bars tagged with the same alphabetic letter do not differ significantly at a $95 \%$ confidence level, while the letters followed by the asterisk symbol $\left({ }^{*}\right)$ denote significantly higher values $(\mathrm{P}<0.05)$

It was observed that after extraction and fractionation with hexane, methylene chloride, and ethyl acetate, the ethyl acetate fraction showed the highest activity (40), indicating slightly polar bioactive metabolites. The ethyl acetate fraction showed antioxidant and antimicrobial activities (41-44). The brine shrimp assay showed that isolates may produce cytotoxic compounds, which corresponds to previous reports on Chaetomium as an endophyte with cytotoxic and antioxidant potential (41, 45-47).

The partially purified ethyl acetate fraction was also evaluated for antimicrobial potential against different clinical MDR isolates (Figure 6). The results indicated that this fraction possesses promising activity against $P$. aeruginosa, S. aureus, Enterococcus sp., and E. coli. The ethyl acetate fraction also showed promising antifungal activity against $C$. albicans, which could be attributed to antifungal azaphilones as reported for the fungus Chaetomium cupreum $(48,49)$. The cultural optimization studies revealed that different media compositions and cultural conditions have significant impacts on the growth and antimicrobial activity of the NF15 strain. On the basis of the results obtained in this study, it is possible to suggest the appropriate media and operational parameters for culturing NF15 in order to isolate and characterize unique and novel biologically active metabolites.

\subsection{Conclusion}

Endophytic fungi are a poorly investigated group of microorganisms that represent an abundant and dependable source of bioactive and chemically novel compounds.
These compounds have the potential for exploitation in a wide variety of medical, agricultural, and industrial applications. In the current study, endophytic fungi isolated from the leaves of $J$. adathoda showed promising antibacterial activity; therefore, the isolation of any bioactive compounds from the endophytic fungi of this medicinal plant may facilitate the product-discovery process.

\section{Acknowledgments}

We are very thankful to Prof. Dr. Mir Ajab Khan (Deparment of Plant Sciences, Quaid-i-Azam University, Islamabad, Pakistan) for his help during plant collection and identification.

\section{Footnote}

Authors' Contribution: Study concept and design: Nighat Fatima, Ihsan-Ul-Haq and Safia Ahmed; acquisition of data: Nighat Fatima, Usman Mukhtar and Muniba Jadoon; analysis and interpretation of data: Nighat Fatima, Usman Mukhtar and Muneer Ahmed Qazi; drafting of the manuscript: Nighat Fatima, Usman Mukhtar, Muneer Ahmed Qazi and Muniba Jadoon; critical revision of the manuscript for important intellectual content: Nighat Fatima, Ihsan-Ul-Haq, Muneer Ahmed Qazi, Muniba Jadoon and Safia Ahmed; administrative, technical, and material support: Safia Ahmed and Ihsan-Ul-Haq; study supervision: Safia Ahmed.

\section{References}

1. Tenguria RK, Khan FN. Distribution of endophytic fungi in leaves of azadirachta indica a. Juss.(Neem) of panchmarhi biosphere reserve. Curr Botany. 2011;2(2):29-7.

2. Zhang JY, Tao LY, Liang YJ, Chen LM, Mi YJ, Zheng LS, et al. Anthracenedione derivatives as anticancer agents isolated from secondary metabolites of the mangrove endophytic fungi. Mar Drugs. 2010;8(4):1469-81. doi: 10.3390/md8041469. [PubMed: 20479985].

3. Strobel GA. Endophytes as sources of bioactive products. Microbes Infect. 2003;5(6):535-44. [PubMed: 12758283].

4. Arnold AE, Maynard Z, Gilbert GS, Coley PD, Kursar TA. Are tropical fungal endophytes hyperdiverse?. Ecol Lett. 2000;3(4):267-74. doi: 10.1046/j.1461-0248.2000.00159.x.

5. Owen NL, Hundley N. Endophytes-the chemical synthesizers inside plants. Sci Prog. 2004;87(Pt 2):79-99. [PubMed: 15782772].

6. Tan RX, Zou WX. Endophytes: a rich source of functional metabolites. Nat Prod Rep. 2001;18(4):448-59. [PubMed: 11548053].

7. Gunatilaka AA. Natural products from plant-associated microorganisms: distribution, structural diversity, bioactivity, and implications of their occurrence. J Nat Prod. 2006;69(3):509-26. doi: 10.1021/np058128n. [PubMed: 16562864].

8. Joseph B, Priya RM. Bioactive compounds from endophytes and their potential in pharmaceutical effect: A review. Am Biochem Mol Biol. 2011;1(3):291-309. doi: 10.3923/ajbmb.2011.291.309. 
9. Strobel G, Daisy B. Bioprospecting for microbial endophytes and their natural products. Microbiol Mol Biol Rev. 2003;67(4):491-502. [PubMed: 14665674].

10. Strobel G, Daisy B, Castillo U, Harper J. Natural products from endophytic microorganisms. J Nat Prod. 2004;67(2):257-68. doi: 10.1021/np030397v. [PubMed: 14987067].

11. Zhang Q, Li HQ, Zong SC, Gao JM, Zhang AL. Chemical and bioactive diversities of the genus Chaetomium secondary metabolites. Mini Rev Med Chem. 2012;12(2):127-48. [PubMed: 22372603].

12. Petrini O. Taxonomy of endophytic fungi of aerial plant tissues. Cambridge Unviersity Press; 1986.

13. Hijri M, Redecker D, Petetot JA, Voigt K, Wostemeyer J, Sanders IR. Identification and isolation of two ascomycete fungi from spores of the arbuscular mycorrhizal fungus Scutellospora castanea. Appl Environ Microbiol. 2002;68(9):4567-73. [PubMed:12200315].

14. Nair R, Kalariya T, Chanda S. Antibacterial activity of some selected indian medicinal flora. Turkish Biol. 2005;29(1):41-7.

15. Kanan GJ, Al-Najar RA. In vitro antifungal activities of various plant crude extracts and fractions against citrus post-harvest disease agent penicillium digitatum. Jor J Biol Sci. 2008;1(3):89-99.

16. Qazi MA, Kanwal T, Jadoon M, Ahmed S, Fatima N. Isolation and characterization of a biosurfactant-producing Fusarium sp. BS-8 from oil contaminated soil. Biotechnol Prog. 2014;30(5):1065-75. doi: 10.1002/btpr.1933. [PubMed: 24850435].

17. Paul NC, Kim WK, Woo SK, Park MS, Yu SH. Fungal endophytes in roots of aralia species and their antifungal activity. Plant Pathol $\mathrm{J}$. 2007;23(4):287-94. doi: 10.5423/ppj.2007.23.4.287.

18. Ihsan ul H, Mirza B, Kondratyuk TP, Park EJ, Burns BE, Marler LE, et al. Preliminary evaluation for cancer chemopreventive and cytotoxic potential of naturally growing ethnobotanically selected plants of Pakistan. Pharm Biol. 2013;51(3):316-28. doi:10.3109/13880209.2012.728612. [PubMed: 23137214].

19. Strobel G, Ford E, Worapong J, Harper JK, Arif AM, Grant DM, et al. Isopestacin, an isobenzofuranone from Pestalotiopsis microspora, possessing antifungal and antioxidant activities. Phytochemistry. 2002;60(2):179-83. [PubMed: 12009322].

20. Kjer J, Debbab A, Aly AH, Proksch P. Methods for isolation of marinederived endophytic fungi and their bioactive secondary products. Nat Protoc. 2010;5(3):479-90. doi: 10.1038/nprot.2009.233. [PubMed 20203665].

21. Shinwari ZK, Khan I, Naz S, Hussain A. Assessment of antibacterial activity of three plants used in pakistan to cure respiratory diseases. Afr J Biotechnol. 2009;8(24):7082-7086.

22. Strobel GA, Daisy BH, Castillo U, Harper J. Novel natural products from rainforest endophytes. Natural Products. Humana Press; 2005. pp. 329-51.

23. Prabavathy D, Nachiyar CV. Study on the antimicrobial activity of aspergillus sp isolated from justicia adathoda. Indian J SciTechnol. 2012;5(9):3317-20. doi:10.17485/ijst/2012/v5i9/30679.

24. Vinu AAK, Jayashankara M. Potentiality of endophytic fungi of Justicia wayanadensis as bioagent against Rhizoctonia solani. Asian J Microbiol Biotechnol Enviro Sci. 2011;13(1):7-9.

25. Gangadevi V, Muthumary J. Endophytic fungal diversity from young, mature and senescent leaves of ocimum basilicum l. With special reference to taxol production. Indian J Sci Technol. 2007;1(1):1-12. doi: $10.17485 / \mathrm{ijst} / 2007 / \mathrm{v1i1} / 29181$.

26. Li H, Xiao J, Gao YQ, Tang JJ, Zhang AL, Gao JM. Chaetoglobosins from Chaetomium globosum, an endophytic fungus in Ginkgo biloba, and their phytotoxic and cytotoxic activities. J Agric Food Chem. 2014;62(17):3734-41. doi: 10.1021/jf500390h. [PubMed: 24708412].

27. Zheng QC, Kong MZ, Zhao Q, Chen GD, Tian HY, Li XX, et al. Chaetoglobosin Y, a new cytochalasan from Chaetomium globosum. Fitoterapia. 2014;93:126-31. doi: 10.1016/j.fitote.2013.12.022. [PubMed: 24418656].

28. Kusari S, Hertweck C, Spiteller M. Chemical ecology of endophytic fungi: origins of secondary metabolites. Chem Biol. 2012;19(7):792-8. doi: 10.1016/j.chembiol.2012.06.004. [PubMed: 22840767].
29. Wang Y, Fang X, An F, Wang G, Zhang X. Improvement of antibiotic activity of Xenorhabdus bovienii by medium optimization using response surface methodology. Microb Cell Fact. 2011;10:98. doi: 10.1186/1475-2859-10-98. [PubMed: 22082189].

30. Gogoi DK, Boruah HPD, Saikia R, Bora TC. Optimization of process parameters for improved production of bioactive metabolite by a novel endophytic fungus fusarium sp. Df2 isolated from taxus wallichiana of north east india. World J Microbiol Biotechnol. 2008;24(1):79-87. doi: 10.1007/s11274-007-9442-3.

31. Bhattacharyya PN, Jha DK. Optimization of cultural conditions affecting growth and improved bioactive metabolite production by a subsurface aspergillus strain tsf 146. Int J App Biol Pharma Technol. 2011;2:133-43.

32. Atalla MM, Zeinab HK, Eman RH, Amani AY, Abeer A. Production of some biologically active secondary metabolites from marine-derived fungus varicosporina ramulosa. Malaysian J microbiol. 2008;4(1):1424.

33. Maria GL, Sridhar KR, Raviraja NS. Antimicrobial and enzyme activity of mangrove endophytic fungi of southwest coast of india. J Agricul technol. 2005;1:67-80.

34. Sanchez S, Bravo V, Castro E, Moya AJ, Camacho F. The influence of $\mathrm{pH}$ and aeration rate on the fermentation of D-xylose by Candida shehatae. Enzyme Microb Technol. 1997;21(5):355-60. [PubMed: 9322374].

35. Zhang HW, Song YC, Tan RX. Biology and chemistry of endophytes. Nat Prod Rep. 2006;23(5):753-71. doi: 10.1039/b609472b. [PubMed: 17003908].

36. Sujatha P, Bapi Raju KV, Ramana T. Studies on a new marine streptomycete BT-408 producing polyketide antibiotic SBR-22 effective against methicillin resistant Staphylococcus aureus. Microbiol Res. 2005;160(2):119-26. doi: 10.1016/j.micres.2004.10.006. [PubMed: 15881828].

37. Mathan S, Subramanian V, Nagamony S. Optimization and antimicrobial metabolite production from endophytic fungi aspergillus terreus kc 582297. Eur J Exp Biol. 2013;3:138-44.

38. Farid MA, el-Enshasy HA, el-Diwany AI, el-Sayed el SA. Optimization of the cultivation medium for natamycin production by Streptomyces natalensis. J Basic Microbiol. 2000;40(3):157-66. doi: 10.1002/15214028(200007)40:3<157::AID-JOBM157>3.0.CO;2-1. [PubMed: 10957957].

39. Pranaw K, Singh S, Dutta D, Chaudhuri S, Ganguly S, Nain L. Statistical Optimization of Media Components for Production of Fibrinolytic Alkaline Metalloproteases from Xenorhabdus indica KB-3. Biotechnol Res Int. 2014;2014:293434. doi: 10.1155/2014/293434. [PubMed: 24864214].

40. Strobel G. Harnessing endophytes for industrial microbiology. Curr Opin Microbiol. 2006;9(3):240-4. doi: 10.1016/j.mib.2006.04.001. [PubMed: 16647289].

41. Huang WY, Cai YZ, Xing J, Corke H, Sun M. A potential antioxidant resource: Endophytic fungi from medicinal plants. Economic Botany. 2007;61(1):14-30. doi: 10.1663/0013-0001(2007)61[14:aparef]2.0.co;2.

42. Kumar S, Kaushik N, Proksch P. Identification of antifungal principle in the solvent extract of an endophytic fungus Chaetomium globosum from Withania somnifera. Springerplus. 2013;2(1):37. doi: 10.1186/2193-1801-2-37. [PubMed: 23519550].

43. Srimathi S, Narayani KSD, Muthumary J. Studies on antimicrobial activities of chaetomium atrobrunneum ames against selected microorganisms. J Experi Sci. 2011;2(5):13-18.

44. Wang FW, Jiao RH, Cheng AB, Tan SH, Song YC. Antimicrobial potentials of endophytic fungi residing in quercus variabilis and brefeldin a obtained from cladosporium sp. World J Microbiol Biotech. 2007;23(1):79-83. doi: 10.1007/s11274-006-9195-4.

45. Bashyal BP, Wijeratne EM, Faeth SH, Gunatilaka AA. Globosumones A-C, cytotoxic orsellinic acid esters from the Sonoran desert endophytic fungus Chaetomium globosum. J Nat Prod. 2005;68(5):724-8. doi: 10.1021/np058014b. [PubMed: 15921417].

46. Selim KA, El-Beih AA, Abdel-Rahman TM, El-Diwany AI. Biological evaluation of endophytic fungus, Chaetomium globosum JN711454, as po- 
tential candidate for improving drug discovery. Cell Biochem Biophys. 2014;68(1):67-82. doi:10.1007/s12013-013-9695-4. [PubMed: 23775636].

47. Yadav M, Yadav A, Yadav JP. In vitro antioxidant activity and total phenolic content of endophytic fungi isolated from Eugenia jambolana Lam. Asian Pac J Trop Med. 2014;7S1:S256-61. doi: 10.1016/S19957645(14)60242-X. [PubMed: 25312132].
Louangsysouphanh S, Soytong K, Isobe M, et al. Antifungal azaphilones from the fungus Chaetomium cupreum CC3003. J Nat Prod 2006;69(6):891-5. doi: 10.1021/np060051v. [PubMed: 16792406].

49. Zhang G, Wang F, Qin J, Wang D, Zhang J, Zhang Y, et al. Efficacy assessment of antifungal metabolites from chaetomium globosum no.05, a new biocontrol agent, against setosphaeria turcica. Biol Control. 2013;64(1):90-8. doi:10.1016/j.biocontrol.2012.10.005. 